Quim. Nova, Vol. 29, No. 6, 1372-1376, 2006

\title{
ESTUDO DE RECUPERAÇÃO DE GLIFOSATO E AMPA DERIVADOS EM SOLO UTILIZANDO-SE RESINAS NACIONAIS
}

\author{
Tomaz Alves de Souza, Marcia Helena de Rizzo da Matta*, Émerson Montagner e Adley Bergson Gonçalves de Abreu \\ Departamento de Química, Centro de Ciências Exatas e Tecnologia, Universidade Federal de Mato Grosso do Sul, CP 549, \\ 79070-900 Campo Grande - MS, Brasil
}

Recebido em 18/8/05; aceito em 31/10/05; publicado na web em 11/8/06

\begin{abstract}
STUDY OF RECOVERY AND STABILITY OF DERIVATIZED GLIPHOSATE AND AMPA IN SOIL USING NATIONAL RESINS. In the present paper we studied the recoveries of glyphosate, $N$-(phosphonomethyl)glycine (GLY) and its major metabolite, (aminomethyl)phosphonic acid (AMPA) in soil using national (Brazilian) ion-exchange resins, derivatization by a mixture of trifluoroacetic anhydride and trifluoroethanol and analyses by GC-MS. The quantification limits were $12 \mathrm{ng} \cdot \mathrm{g}^{-1}$ for both compounds and the methodology showed a range of recuperation from 85 to $94 \%$ with coefficients of variation (CV) ranging from 4.07 to $6.91 \%$ for GLY. For AMPA, the mean recoveries ranged from 87 to $102 \%$ with CVs ranging from 5.81 to $6.99 \%$. Additional studies showed that, due to the instability of the derivatized compounds, they must be analysed keeping constant time between derivatization and analysis, preferably less than $24 \mathrm{~h}$.
\end{abstract}

Keywords: glyphosate; AMPA; GC/MS.

\section{INTRODUÇÃO}

No Brasil, o cerrado é uma região muito diferenciada, pois associa grande biodiversidade a uma aparência árida decorrente, em grande parte, de solos pobres e ácidos e da ocorrência de apenas duas estações climáticas: uma seca e outra chuvosa. O relevo, predominantemente plano, facilita o uso de máquinas agrícolas que desmatam rapidamente grandes extensões de área natural. É uma região que abriga vasta diversidade de espécies e importantes bacias hidrográficas, motivo pelo qual sua rápida ocupação torna-se preocupante, podendo gerar impactos ambientais irreversíveis ${ }^{1}$. Uma das principais ameaças à biodiversidade do cerrado é a monocultura intensiva de grãos ${ }^{2}$, onde a soja, com seus insumos, representa a principal cultura em área plantada (6.391.013 ha), totalizando $46,67 \%$ de representação no Brasil ${ }^{3}$.

$\mathrm{O}$ glifosato, $\mathrm{N}$-(fosfonometil)-glicina, GLI, é um herbicida não seletivo, sistêmico, pós-emergente, representando atualmente $60 \%$ do mercado mundial de herbicidas não seletivos. Sua elevada eficiência na eliminação de ervas daninhas e o fato de possuir uma baixa toxicidade aos que o manipulam são responsáveis pelo seu grande sucesso $^{4}$. Na região do cerrado brasileiro, figura entre os principais agrotóxicos utilizados na cultura da soja, com uma quantidade aproximada de 0,5 a $1,0 \mathrm{~kg} / \mathrm{ha}$ ou L/ha ${ }^{2}$. O GLI é classificado como uma glicina substituída e também como um herbicida organofosforado, sem, no entanto, afetar o sistema nervoso da mesma maneira que outros organofosforados (em geral inseticidas inibidores da enzima acetilcolinesterase) e, apesar de ser citado como pouco tóxico, há evidência de efeitos deletérios no ambiente após seu uso prolongado, principalmente devido à resistência adquirida por algumas espécies de ervas ${ }^{4}$. Adicionalmente, é necessário atentar-se para outro risco, visto que a formulação mais comercializada no País contém um surfactante com significativa ação irritativa dermatológica ${ }^{6}$, conhecido como POEA (polietoxietileno amina) ${ }^{7}$.

O modo primário de ação do GLI é a inibição competitiva da 5enolpiruvilchiquimato-3-fosfato sintase (EPSPS), interferindo na produção de aminoácidos aromáticos e outros compostos aromáti-

*e-mail: marciarm@nin.ufms.br cos em plantas, as quais, quando tratadas com este herbicida, não conseguem produzir os aminoácidos aromáticos necessários para sua sobrevivência ${ }^{4}$.

O ácido aminometilfosfônico (AMPA) é o principal metabólito do GLI, decorrente da sua degradação por ação microbiológica 8,9 . Embora tenha toxicidade baixa (sua $\mathrm{DL}_{50}$ é de $8.300 \mathrm{mg} \mathrm{kg}^{-1}$ ), é mais persistente que o GLI. Estudos estimam sua meia-vida em solo entre 119 e 958 dias enquanto que pesquisadores encontraram tempos de meia-vida inferiores a 3 dias para o $\mathrm{GLI}^{10}$. Por isso, torna-se importante o desenvolvimento de métodos de extração e análise tanto para GLI como para AMPA.

Devido à alta polaridade do GLI e sua tendência em formar espécies iônicas, a maioria dos procedimentos de extração do herbicida baseiam-se em reações ácido-base, onde o composto, inicialmente ligado a espécies iônicas do solo através do grupo fosfato, passa, então, a interagir com os íons da solução. Desta forma, nos processos de extração, são utilizadas, geralmente, soluções de bases fortes, sais de bases fortes, bases fracas ou ácidos fracos, seguindo-se, na maioria das vezes, de etapas de retenção/eluição em resinas de troca iônica ${ }^{11}$. A eficiência do processo de extração do GLI e do AMPA está diretamente relacionada ao tipo de solo. Em geral, as melhores recuperações são observadas em solos com alto teor de matéria orgânica, enquanto que para solos argilosos e minerais, devido à elevada competitividade pelos sítios iônicos do solo, a eficiência do processo de extração fica comprometida, resultando em menores recuperações ${ }^{12}$. A utilização da cromatografia líquida de alta eficiência (CLAE) com detector de UVvisível na determinação do GLI e seus metabólitos não é apropriada, devido ao fato deste herbicida não possuir em sua molécula agentes cromóforos observáveis satisfatoriamente neste detector, o que dificulta a identificação e quantificação; entretanto, a utilização desta mesma técnica com detector de fluorescência, seguida da derivação pós-coluna do GLI em um composto fluorescente, tem sido utilizada ${ }^{13}$, sendo este o procedimento recomendado pela Agencia de Proteção Ambiental dos Estados Unidos (EPA) ${ }^{14}$. A técnica de cromatografia gasosa de alta resolução (CGAR), utilizando detectores específicos ou acoplada à espectrometria de massas (CG-EM), também pode ser empregada para determinação de GLI e seus metabólitos ${ }^{15-18}$; porém, para este tipo de determinação, faz-se necessária uma prévia derivação para obtenção 
de compostos voláteis, visto que os analitos apresentam elevada polaridade e pouca volatilidade, o que faz com que o processo de preparação da amostra seja demorado e trabalhoso ${ }^{4}$. Várias outras técnicas analíticas para determinação de GLI e AMPA foram descritas, tais como cromatografia em camada delgada ${ }^{19}$, polarografia ${ }^{20}$, ressonância magnética nuclear de ${ }^{31} \mathrm{P}^{21}$, espectrofotometria ${ }^{22}$ e eletroforese ${ }^{23}$.

Quanto à derivação, o GLI pode ser considerado um caso especial, devido à sua natureza polar e alta solubilidade em água, o que limita a possibilidade de se usar técnicas padrão de derivação, empregadas freqüentemente para análise posterior por $\mathrm{CG}^{24}$. $\mathrm{O}$ uso de misturas de anidridos fluorados e álcoois perfluorados fornece derivados do GLI e AMPA, que podem ser detectados por CG-EM com sensibilidade e seletividade elevadas ${ }^{17}$. Geralmente, o processo envolve o uso de anidrido trifluoroacético (TFAA) e trifluoroetanol (TFE) $)^{15,25}$, TFAA e diazometano ${ }^{26}$, e ácido heptafluorobutírico (HFBA) e 2-cloroetanol ${ }^{27}$, sendo que no primeiro caso, a reação de derivação é feita com excesso de TFAA e TFE devido ao fato de que as etapas de formação dos compostos derivados são de equilíbrio e, com este excesso, a reação deslocar-se-á no sentido de formar compostos derivados, como mostra a Figura 1.

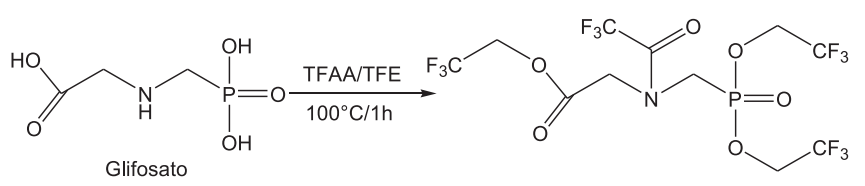

Glifosato-Derivado

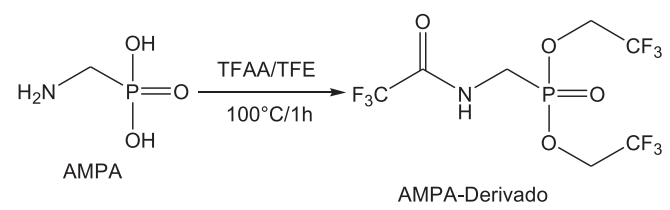

Figura 1. Esquema simplificado de conversão do GLI e AMPA em seus respectivos derivados

A presente pesquisa propôs-se avaliar um procedimento de derivação do GLI e AMPA com TFAA e TFE, a partir de amostras de solo, utilizando-se, nas etapas preliminares de purificação, resinas trocadoras de íons de fabricação nacional, por serem de custo bem inferior às importadas, comumente utilizadas neste processo. Baseando-se em uma pesquisa que utilizou os mesmos reagentes ${ }^{18}$, procedeu-se às análises cromatográficas dos derivados no período máximo de uma semana. Adicionalmente, devido aos derivados obtidos serem citados como pouco estáveis, e não existirem dados na literatura sobre esta questão, optou-se por avaliar também a estabilidade dos compostos derivados em dois tipos de armazenamento em função do tempo decorrido após as derivações. Uma vez que tais parâmetros influenciam diretamente nos resultados obtidos, o estudo aqui realizado é de grande importância, pois uma análise efetuada, utilizando-se os derivados de GLI e AMPA apresentados na Figura 1, sem o conhecimento da estabilidade destes compostos, pode levar a resultados imprecisos.

\section{PARTE EXPERIMENTAL}

\section{Reagentes, soluções e materiais}

Os padrões de GLI (98\%) e AMPA (10 ng $\left.\mu \mathrm{L}^{-1}\right)$, ambos certificados, foram adquiridos de Dr. Ehrenstorfer GmbH (Augsburg,
Germany). Foi preparada solução estoque de $1009 \mathrm{mg} \mathrm{L}^{-1}$ de GLI em água deionizada, solução intermediária de GLI com concentração de $100 \mathrm{mg} \mathrm{L}^{-1}$ e soluções de trabalho de $1 \mathrm{mg} \mathrm{L}^{-1}$, tanto para GLI quanto para AMPA.

Na extração do GLI e AMPA do solo foi utilizada solução (1,0 mol L $\mathrm{L}^{-1}$ ) de $\mathrm{NaOH}$ (Dinâmica, 97\%) e no processo de purificação com resinas trocadoras de íons, Amberlite IRA-420 (Cl-) e Amberlite IR-120 $\left(\mathrm{Na}^{+}\right)$, ambas da Synth, utilizou-se como eluente solução (6,0 mol L-1) de $\mathrm{HCl}(36,9 \%$, Mallinckrodt); como solventes, foram utilizados o metanol $(99,5 \%)$ da Science e acetato de etila $(99,9 \%)$ da Merck, e água ultra-purificada (Milli-Q $\left.{ }^{\circledR}\right)$. Os reagentes anidrido trifluoroacético (TFAA) (99,5\%), da Vetec, e trifluoroetanol (TFE) (99\%), da J. T. Baker, foram utilizados na derivação.

\section{Coleta, caracterização e preparação das amostras}

Para os estudos de recuperação, amostras simples de solo foram coletadas na profundidade de $0-10 \mathrm{~cm}$ no campus da Universidade Federal de Mato Grosso do Sul, em área de Reserva Particular do Patrimônio Natural (RPPN); sendo a amostra composta armazenada em sacos de polietileno em local seco, sob abrigo de luz. Amostras de laboratório foram caracterizadas pelo IAGRO, Departamento de Inspeção e Defesa Agropecuária de Mato Grosso do Sul. As amostras foram peneiradas em malha de $2 \mathrm{~mm}$ e secas ao ar. Foram determinadas as umidades gravimétrica e residual do solo utilizado ${ }^{28}$.

Amostras, contendo $10 \mathrm{~g}$ do solo, foram fortificadas com 12, 36 e $60 \mathrm{ng} \mathrm{g}^{-1}$ de GLI e AMPA e, após um período de aproximadamente $24 \mathrm{~h}$, foram submetidas ao processo de extração do GLI e AMPA.

\section{Extração do GLI e AMPA}

As amostras foram tratadas por $30 \mathrm{~min}$ com $25 \mathrm{~mL}$ de $\mathrm{NaOH}$ (1,0 $\left.\mathrm{mol} \mathrm{L}^{-1}\right)$ em ultra-som, sendo posteriormente centrifugadas por $10 \mathrm{~min}$ em centrífuga (Cole-Parmer) a $3400 \mathrm{rpm}$.

O sobrenadante de cada amostra foi filtrado em papel de filtro qualitativo, sendo adicionada a cada extrato uma alíquota de 4,2 $\mathrm{mL}$ de $\mathrm{HCl}$ concentrado, ajustando-se o $\mathrm{pH}$ para 2 e o volume completado com água ultrapura até $200 \mathrm{~mL}$. As amostras foram mantidas à temperatura ambiente por um período de $3 \mathrm{~h}$ para que ocorresse a decantação de partículas que se encontravam em suspensão. Retirou-se uma alíquota de $50,0 \mathrm{~mL}$ da parte superior de cada extrato para purificação.

\section{Purificação com resinas trocadoras de íons}

Colunas de vidro com $1,5 \mathrm{~cm}$ de diâmetro e $18 \mathrm{~cm}$ de comprimento foram empacotadas com $8,0 \mathrm{~mL}$ de resina catiônica, Amberlite IR-120. A resina foi condicionada com a adição seqüencial de 20,0 $\mathrm{mL}$ de água deionizada, $40,0 \mathrm{~mL}$ de $\mathrm{HCl}\left(0,2 \mathrm{~mol} \mathrm{~L}^{-1}\right)$ e $1,0 \mathrm{~mL}$ de $\mathrm{HCl}\left(6,0 \mathrm{~mol} \mathrm{~L}^{-1}\right)$, descartando-se todo o eluato. As alíquotas de 50,0 $\mathrm{mL}$ dos extratos foram adicionadas nas colunas e eluídas com $1 \times 2,8$ $\mathrm{mL}$ e $2 \times 3,7 \mathrm{~mL}$ de $\mathrm{HCl}\left(6,0 \mathrm{~mol} \mathrm{~L}^{-1}\right)$ sob fluxo de $2 \mathrm{~mL} \mathrm{~min}{ }^{-1}$, repetindo-se o procedimento. Os eluatos foram coletados em recipientes apropriados, sendo a eles adicionados $6,0 \mathrm{~mL}$ de $\mathrm{NaOH}(10,0$ mol L-1) e o pH ajustado na faixa de 6-8.

Outras colunas de vidro, com as mesmas especificações das anteriores, foram empacotadas com 4,0 mL de resina aniônica, Amberlite IRA-420, sendo condicionadas com $3 \times 2,5 \mathrm{~mL}$ de $\mathrm{HCl}$ (6,0 $\left.\mathrm{mol} \mathrm{L}^{-1}\right)$ e $1,0 \mathrm{~mL}$ de $\mathrm{HCl}$ concentrado, descartando-se todo o eluato. Os eluatos tratados com a resina catiônica foram adicionados a estas colunas sob fluxo de $2 \mathrm{~mL} \mathrm{~min}^{-1}$, repetindo-se este procedimento. As substâncias retidas nesta coluna foram eluídas com $1 \times 1,0 \mathrm{~mL}$ e $2 \times 2,0 \mathrm{~mL}$ de $\mathrm{HCl} 6,0 \mathrm{~mol} \mathrm{~L}^{-1}$. As amostras foram 
coletadas em balões de fundo redondo e levadas à secura em evaporador rotativo (TE-120, Tecnal) a $60{ }^{\circ} \mathrm{C}$ e pressão de -500 $\mathrm{mmHg}$. Em seguida, adicionou-se a cada uma delas 5,0 $\mathrm{mL}$ de água purificada e evaporou-se à secura novamente. Retomou-se o volume de cada amostra para 1,0 mL, com uma mistura águametanol-HCl (160-40-2,7) (v/v/v), para impedir que o GLI e o AMPA ficassem adsorvidos nas paredes dos balões, sendo então transferidas para ampolas de vidro.

\section{Derivação e análise cromatográfica das amostras}

As amostras contidas nas ampolas de vidro, do item anterior, foram evaporadas à secura sob fluxo de nitrogênio, e adicionados $800 \mu \mathrm{L}$ de TFAA e $400 \mu \mathrm{L}$ de TFE. Após lacrarem-se as ampolas, estas foram colocadas em estufa a $100{ }^{\circ} \mathrm{C}$, por $1 \mathrm{~h}$. Após atingirem a temperatura ambiente, secou-se novamente sob fluxo de nitrogênio e retomou-se as amostras a $1,0 \mathrm{~mL}$ com acetato de etila, sendo então encaminhadas para a análise cromatográfica, utilizando-se o modo de monitoramento de íons selecionados (modo SIM). Cada amostra foi injetada 5 vezes.

Para construção das curvas analíticas, utilizou-se, para a derivação, a adição de padrões nas concentrações de 30, 60, 90, 150 e $300 \mu \mathrm{g} \mathrm{L}^{-1}$ de AMPA e GLI diretamente nas ampolas, as quais foram secas sob fluxo de nitrogênio. Em seguida, efetuou-se a adição $800 \mu \mathrm{L}$ de TFAA e $400 \mu \mathrm{L}$ de TFE e, depois de lacradas as ampolas, estas foram colocadas em estufa a $100{ }^{\circ} \mathrm{C}$, por $1 \mathrm{~h}$. Após atingirem a temperatura ambiente e serem abertas, foram secas novamente sob fluxo de nitrogênio e o volume das mesmas retomado a $1,0 \mathrm{~mL}$ com acetato de etila, reservando-se então, sob refrigeração, até o momento da análise. Cada amostra foi injetada 7 vezes no modo SIM.

\section{Avaliação da estabilidade do GLI e AMPA derivados}

Amostras, em triplicatas, derivadas de $250 \mu \mathrm{g} \mathrm{L}^{-1}$ de GLI e AMPA foram divididas em duas alíquotas, sendo uma armazenada em freezer $\left(-2^{\circ} \mathrm{C}\right)$ e outra em geladeira $\left(5^{\circ} \mathrm{C}\right)$. Acompanhou-se a degradação dos compostos derivados, via área de integração no CG/EM, por um período de 15 dias nos dois meios de armazenamento.

\section{Equipamento}

As análises foram realizadas em um cromatógrafo a gás modelo GC-17A com detector de massa QP-5000, da Shimadzu, equipado com uma coluna capilar VA-608 da Varian de $30 \mathrm{~m}$ de comprimento, 0,32 $\mathrm{mm}$ de diâmetro interno e $0,5 \mu \mathrm{m}$ de espessura do filme. Foi injetado $1 \mu \mathrm{L}$ de cada amostra no modo sem divisão de amostra ("splitless"). A temperatura do injetor foi de $260^{\circ} \mathrm{C}$, a da interface foi de $260^{\circ} \mathrm{C}$ e a da coluna inicialmente foi de $70^{\circ} \mathrm{C}$ por 2 min, em seguida foi elevada para $270{ }^{\circ} \mathrm{C}\left(28,8^{\circ} \mathrm{C} \mathrm{min}{ }^{-1}\right)$, sendo esta última mantida por $6 \mathrm{~min}$. A pressão na coluna foi de $20 \mathrm{kPa}$ e o fluxo de $1,5 \mathrm{~mL} \mathrm{~min}^{-1}$, utilizando-se como gás de arraste hélio ultrapuro (White Martins). O espectrômetro de massas foi operado na modalidade de impacto de elétrons (EI). Os íons monitorados foram m/z 238, 260, 411 para o GLI e m/z 246 e 302 para o AMPA.

\section{RESULTADOS E DISCUSSÃO}

\section{Análise do solo}

O conhecimento das propriedades físico-químicas do solo é de suma importância em análises de pesticidas, pois fatores como $\mathrm{pH}$, teores de argila e matéria orgânica podem influenciar de forma significativa nas recuperações dos analitos. Na Tabela 1 estão os dados referentes à análise do solo utilizado, efetuada pelo IAGRO.

Conforme a Tabela 1, o solo, proveniente de área de cerrado nativo, foi classificado como argiloso, pois tem teor de argila maior que $35 \%$.

A interpretação de teores de micronutrientes no solo é assunto polêmico no âmbito de pesquisadores de solos, não havendo ainda um consenso sobre o tema que facilite o entendimento. As culturas, diferentes tipos de manejos e classes de solos são fortes complicadores. Os teores na planta são mais consensuais e de uso geral na agronomia, pois a planta reflete melhor a disponibilidade dos mesmos. O ferro é um elemento que ora aparece nas várias tabelas de interpretação, ora não. Este é um elemento abundante em nossos solos, com poucos problemas de deficiência, e apresenta dificuldades na determinação/interpretação, pois é muito influenciado pelo estado de óxido-redução do meio.

A literatura pesquisada ${ }^{29}$ classifica os teores encontrados de $\mathrm{Fe}$, $\mathrm{Mn}, \mathrm{Zn}$ e $\mathrm{Cu}$ como altos, o que é característico para solos de cerra$\mathrm{do}^{2}$. No entanto, o teor de $\mathrm{Al}$ é classificado como baixo, o que não é comum neste tipo de solo; porém, a literatura ${ }^{30}$ apresenta resultados de análise de solo coletados na mesma região com teores de alumínio também muito baixos.

Um dos primeiros trabalhos ${ }^{8}$ sobre adsorção de GLI a íons minerais concluiu que o herbicida é mais facilmente adsorvido em solos argilosos que em arenosos por íons metálicos na seguinte ordem $\mathrm{Ca}^{2+}<\mathrm{Mn}^{2+}<\mathrm{Zn}^{2+}<\mathrm{Mg}^{2+}<\mathrm{Fe}^{3+}<\mathrm{A}^{3+}$ sendo, portanto possível a adsorção do GLI na matriz estudada devido aos altos teores de alguns destes íons. Adicionalmente, a quantidade considerável de cobre (II) também pode ocasionar a complexação do GLI, visto que a presença deste íon aumenta a adsorção do herbicida ao solo, devido à maior habilidade do complexo formado de ser adsorvido se comparado com o GLI livre ${ }^{31}$. Como o teor de fósforo é muito baixo, isto sugere que não há competição significativa entre o GLI e os íons fosfato pelos sítios de adsorção específica do solo ${ }^{32}$.

\section{Avaliação do método}

O método descrito constitui uma alternativa eficaz para a determinação de GLI e AMPA em solo com a vantagem, sobre outros métodos similares, de usar resinas de menor custo, produzidas no Brasil. A pesquisa do GLI em diversas matrizes é de grande importância devido a este herbicida ser o mais vendido no mundo e, também, devido à liberação do plantio da soja transgênica no nosso País, a qual é resistente ao GLI, o que pode acarretar um uso indiscriminado deste herbicida.

A análise cromatográfica por CG-EM apresentou boa seletividade, como pode ser observado na Figura 2.

Os limites de detecção (LD) e de quantificação (LQ) do equipa-

Tabela 1. Análise do solo utilizado

\begin{tabular}{|c|c|c|c|c|c|c|c|c|c|c|c|c|c|}
\hline \multirow[t]{2}{*}{$\overline{\mathrm{pH}}$} & \multirow[t]{2}{*}{ Textura } & \multirow{2}{*}{$\begin{array}{c}\text { M. O. } \\
\%\end{array}$} & \multirow{2}{*}{$\begin{array}{c}\mathrm{P} \\
\mathrm{mg} / \mathrm{cm}^{3}\end{array}$} & \multirow{2}{*}{$\begin{array}{c}\mathrm{K} \\
\mathrm{mg} / \mathrm{dm}^{3}\end{array}$} & \multirow{2}{*}{$\begin{array}{c}\mathrm{Fe} \\
\mathrm{mg} / \mathrm{dm}^{3}\end{array}$} & \multirow{2}{*}{$\begin{array}{c}\mathrm{Mn} \\
\mathrm{mg} / \mathrm{dm}^{3}\end{array}$} & \multirow{2}{*}{$\begin{array}{c}\mathrm{Zn} \\
\mathrm{mg} / \mathrm{dm}^{3}\end{array}$} & \multirow{2}{*}{$\begin{array}{c}\mathrm{Al} \\
\mathrm{mg} / \mathrm{dm}^{3}\end{array}$} & \multirow{2}{*}{$\begin{array}{l}\mathrm{Ca}+\mathrm{Mg} \\
\mathrm{mg} / \mathrm{dm}^{3}\end{array}$} & \multirow{2}{*}{$\begin{array}{c}\mathrm{Cu} \\
\mathrm{mg} / \mathrm{dm}^{3}\end{array}$} & \multicolumn{3}{|c|}{ Granulometria (\%) } \\
\hline & & & & & & & & & & & Argila & Silte & Areia \\
\hline 5,7 & 1 & 1,5 & 2,3 & 0,19 & 110,28 & 141,00 & 37,17 & 0,2 & 2,6 & 22,16 & 44 & 20 & 36 \\
\hline
\end{tabular}




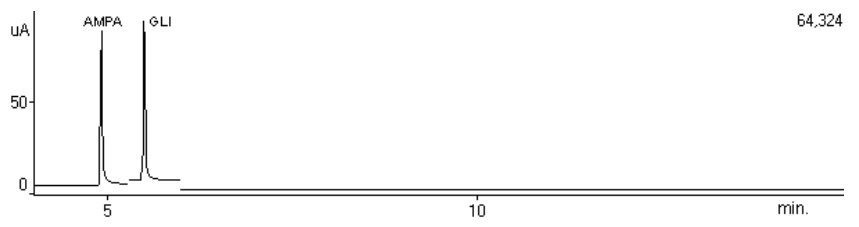

Figura 2. Cromatograma de AMPA e GLI

mento foram calculados através da relação sinal/ruído de 3 e 10 vezes, respectivamente. Os valores de LD e LQ, relativos ao equipamento nas condições analíticas utilizadas, foram, para ambos GLI e AMPA, de 10 e $30 \mathrm{ng} \mathrm{mL}^{-1}$, respectivamente. Os valores de LD e LQ relativos ao método foram, respectivamente, de 4 e $12 \mathrm{ng}$ $\mathrm{g}^{-1}$, tanto para GLI quanto para AMPA. Indicando, assim, que o método é adequado para detectar e quantificar o herbicida e seu metabólito em baixos níveis de concentração.

As curvas analíticas do GLI e AMPA mostraram boa linearidade e coeficientes de correlação significativos, como se observa na Tabela 2. O intervalo linear de trabalho foi de 30 a $300 \mu \mathrm{g} \mathrm{L}^{-1}$ para GLI e AMPA.

Tabela 2. Curvas analíticas para GLI e AMPA

\begin{tabular}{lcc}
\hline Composto & Curva analítica & Coeficiente de correlação (r) \\
\hline GLI & $y=345,35 x-2481,22$ & 0,99874 \\
AMPA & $y=272,45 x-236,97$ & 0,99955 \\
\hline
\end{tabular}

As amostras, fortificadas, em triplicatas, em três níveis de concentração (GLI e AMPA)- baixo, médio e alto- foram analisadas também em triplicada, sendo os dados obtidos exibidos na Tabela 3. Observa-se pela Tabela 3 que o método empregado possui uma boa precisão, pois o coeficiente de variação percentual é inferior a $15 \%$ em todos os níveis de fortificação.

Tabela 3. Eficiência da recuperação obtida, para GLI e AMPA

\begin{tabular}{lcccc}
\hline \multirow{2}{*}{$\begin{array}{l}\text { Nível de fortificação } \\
\left(\mathrm{ng} \mathrm{g}^{-1}\right)\end{array}$} & \multicolumn{2}{l}{ Recuperação (\%) } & \multicolumn{2}{c}{ C.V. (\%) } \\
& GLI & AMPA & GLI & AMPA \\
\hline 12 & 85 & 87 & 6,91 & 5,81 \\
36 & 88 & 91 & 6,07 & 6,99 \\
60 & 94 & 102 & 4,07 & 6,33 \\
\hline
\end{tabular}

As recuperações variaram entre $85-94 \%$ para o GLI e 87-102\% para AMPA, sendo consideradas como resultados excelentes, visto que se encontram na faixa de recuperação, entre 70$120 \%$, recomendada pela $\mathrm{EPA}^{14}$. O método apresentou boas recuperações, mesmo se utilizando amostras de solo com significativos teores de minerais.

As resinas trocadoras de íons mostraram-se tão ou mais eficientes no processo de purificação que suas similares Chelex 100 e AG 1-X8 utilizadas em um trabalho com metodologia de análise semelhante ao descrito nesse $\operatorname{artigo~}^{18}$. Isso pode ser observado comparando-se os resultados de recuperação, pois além dos teores de minerais presentes no solo, as resinas também influenciam diretamente no resultado da análise pois, se a troca iônica entre a resina e GLI e AMPA não for suficientemente eficiente, ocorrerão perdas do analito nesta etapa e a análise ficará comprometida. As resinas nacionais mostram-se uma excelente alternativa às resinas importadas, devido a terem uma eficiência muito boa, com a vantagem de terem um custo muito menor.

\section{Avaliação da estabilidade dos derivados de GLI e AMPA}

As Figuras 3 e 4, a seguir, são relativos à avaliação da estabilidade do GLI e do AMPA derivados nos dois meios de armazenamento, freezer $\left(-2^{\circ} \mathrm{C}\right)$ e geladeira $\left(5^{\circ} \mathrm{C}\right)$.

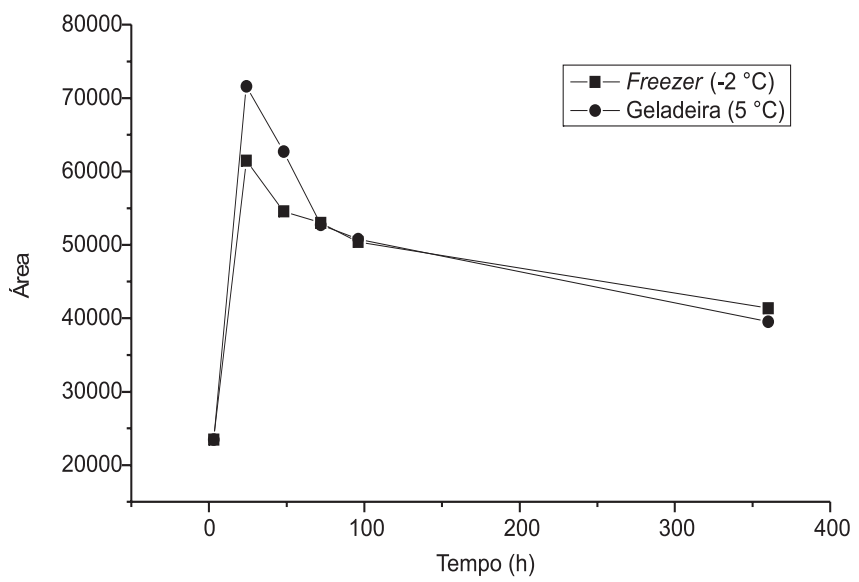

Figura 3. Acompanhamento da degradação do GLI derivado nos dois meios de armazenamento

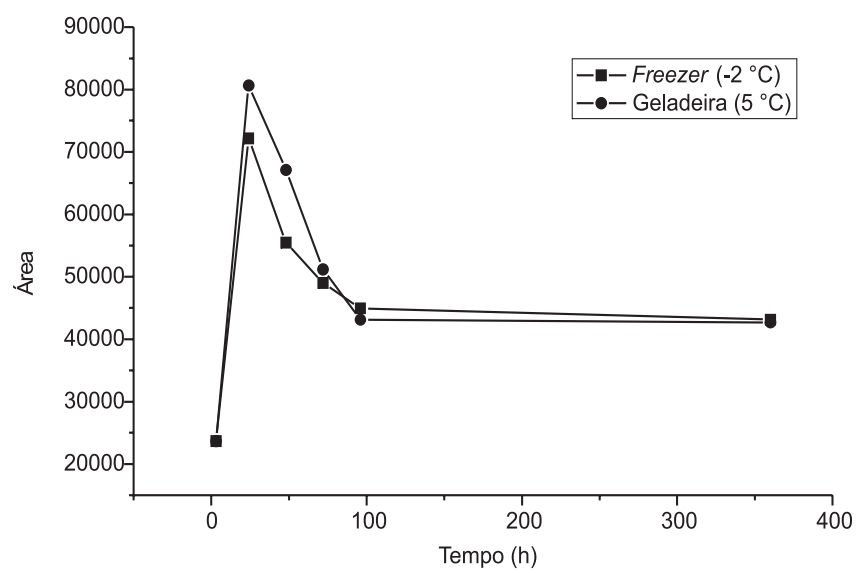

Figura 4. Acompanhamento da degradação do AMPA derivado nos dois meios de armazenamento

Tanto GLI como AMPA apresentam picos com áreas maiores após passadas $24 \mathrm{~h}$ da derivação com TFAA e TFE, não importando onde fiquem armazenados. Isso pode ser devido ao fato de que a reação demore um determinado intervalo de tempo para se deslocar no sentido do composto derivado, com possíveis influências do solvente utilizado, bem como da temperatura, sendo, portanto, necessários estudos cinéticos neste sentido. A dependência da temperatura pode ser observada nas Figuras 3 e 4, onde a área obtida para os derivados é maior quando estes são armazenados a uma temperatura maior, 5 ${ }^{\circ} \mathrm{C}$ (geladeira), que quando são armazenados a $-2{ }^{\circ} \mathrm{C}$ (freezer).

Aplicando-se o Teste $t$ de Student sobre as médias dos resultados obtidos, pode-se afirmar que, para o armazenamento a um período maior que uma semana, não há diferença significativa com $95 \%$ de confiança entre as áreas de GLI e AMPA derivados nos dois tipos de armazenamento, embora haja diminuição na área dos picos devido à degradação dos derivados. Porém, o pico de maior área (após $24 \mathrm{~h}$ ), tanto para GLI derivado quanto para AMPA derivado, é maior quando estes são armazenados em geladeira. Portanto, para se obter melhores resultados com o método proposto, as amostras de- 
vem ser armazenadas em geladeira por 24 h, quando então devem ser imediatamente analisadas.

\section{CONCLUSÕES}

O método de determinação de GLI e AMPA por CG-EM proposto mostrou-se bastante eficiente, com boa linearidade, precisão e reprodutibilidade. As recuperações foram satisfatórias mesmo utilizando-se um solo com altos teores de minerais, os quais podem interferir na análise. Acredita-se também que esta mesma metodologia possa ser utilizada de forma eficiente em solos com baixos teores de minerais. O processo de purificação dos extratos, com as resinas trocadoras de íons Amberlite IR-120 e Amberlite IRA-420, mostrou-se eficaz, indicando ser esta técnica mais econômica que outras indicadas na literatura, as quais aumentam significantemente o custo desta etapa da análise.

Quanto à estabilidade, sugere-se que os derivados neste tipo de estudo sejam analisados cromatograficamente após exatamente o mesmo período decorrido após o término do processo de derivação, de preferência em 24 h, para que não haja diferença nos resultados devido à instabilidade dos derivados.

\section{AGRADECIMENTOS}

Ao apoio da UFMS, do CNPq, da CAPES, da FUNDECT/MS e do IAGRO/MS.

\section{REFERÊNCIAS}

1. http://www.eco.unicamp.br/ecoeco/artigos/encontros/downloads/mesa2/ 4.pdf, acessada em Fevereiro 2005.

2. Oliveira-Filho, E. C.; Lima, J. E. F. W.; Potencial de Impacto da Agricultura sobre os Recursos Hídricos na Região do Cerrado, EMBRAPA-CPAC: Planaltina, 2002, Documentos 56.

3. Garagorry, F. L.; Rego, A. M.; AGROTEC: base relacional de dados de estatísticos. Estrutura de dados, EMBRAPA: Brasília, 1997; Chaib Filho, H.; Garagorry, F. L.; Junqueira, N.; Anais do $17^{\circ}$ Congresso Brasileiro de Fruticultura, Belém, Brasil, 2002; Garagorry, F. L.; Chaib Filho, H.; Junqueira, N.; Anais do $17^{\circ}$ Congresso Brasileiro de Fruticultura, Belém, Brasil, 2002.

4. Amarante Jr., O. P.; Santos, T. C. R.; Brito, N. M.; Ribeiro, M. L.; Quim. Nova 2002, 25, 589.

5. http://www.nortox.com.br/arquivos/pdf/pdf_bula_13.pdf, acessada em Outubro 2005.

6. http://www.agr.unicamp.br/tomates/pdfs/eftoxic.pdf, acessada em Fevereiro 2005.

7. Gehin, A.; Guillaume, Y. C.; Millet, J.; Guyon, C.; Nicod, L.; Int. J. Pharm. 2005, 288, 219.

8. Sprankle, P.; Meggitt, W. F.; Penner, D.; Weed Sci. 1975, 23, 229.

9. Rueppel, M. L.; Brightwell, B. B.; Schaefer, J.; Marvel, J. T.; J. Agric. Food Chem. 1977, 30, 517; Zaranyika, M. F.; Nyandoro, M. G.; J. Agric. Food Chem. 1993, 41, 838.
10. http://www.aracruz.com/microbacia/shared/relatorio_1997.pdf, acessada em Outubro 2005.

11. Amarante Jr., O. P.; Santos, T. C. R.; Brito, N. M.; Ribeiro, M. L.; Quim. Nova 2002, 25, 420 .

12. Thompson, D. G.; Cowell, J. E.; Daniels, R. J.; Staznik, B.; Macdonald, L. M.; J. AOAC Int. 1989, 72, 355.

13. Miles, C. J.; Leong, G.; LCGC North Am. 1992, 10, 452; Abdullah, M. P.; Daud, J.; Hong, K. S.; Yew, C. H.; J. Chromatogr., A 1995, 697, 363.

14. Environmental Protection Agency (EPA); Drinking Water Methods From Methods for the Determination of Organic Compounds in Drinking Water, Supplement I, EPA/600/4-90/020. Methods and Guidance for Analysis of Water, 1990.

15. Deyrup, C. L.; Chang. S.; Weintraub, R. A.; Moye, A.; J. Agric. Food Chem. $\mathbf{1 9 8 5}, 33,944$.

16. Roy, D. N.; Konar, S. K.; J. Agric. Food Chem. 1989, 37, 441; Eberbach, P. L.; Douglas, L. A.; J. Agric. Food Chem. 1991, 39, 1776; Tsunoda, N.; J. Chromatogr., A 1993, 637, 167; Kataoka, H.; Ryu, S.; Sakiyama, N.; Makita, M.; J. Chromatogr., A 1996, 726, 253; Stalikas, C. D.; Konidari, C. N.; J. Chromatogr., A 2001, 907, 1; Kudzin, Z. H.; Gralak, D. K.; Drabowicz, J.; Luczak, J.; J. Chromatogr., A 2002, 947, 129; Kudzin, Z. H.; Gralak, D. K.; Andrijewski, G.; Drabowicz, J.; Luczak, J.; J. Chromatogr., A 2003, 998, 183.

17. Alferness, P. L.; Iwata, Y.; J. Agric. Food Chem. 1994, 42, 2751.

18. Börjesson, E.; Torstensson, L.; J. Chromatogr., A 2000, 886, 207.

19. Young, J. C.; Khan, S. V.; Marriage, P. B.; J. Agric. Food Chem. 1977, 25, 918; Bunyatyan, Y. O. A.; Gevorgyan, A.; A. Gig. Sanit. 1984, 5, 43.

20. Friestad, H. O.; Bronstad, J. O.; J. AOAC Int. 1985, 68, 76.

21. Dickson, S. J.; Meinhold, R. H.; Beer, I. D.; Koelmeyer, T. D.; J. Anal. Toxicol. 1988, 12, 284.

22. Daniele, P. G.; De Stefano, C.; Prenesti, E.; Sammartano, S.; Talanta 1997, 45, 425; Zhemchuzhin, S. G.; Gorobets, R. P.; Zh. Anal. Khim. 1989, 44, 741.

23. Cikalo, M. G.; Goodall, D. M.; Matthews, W.; J. Chromatogr., A 1996, 745, 189; Chang, S. Y.; Liao, C.; J. Chromatogr., A 2002, 959, 309; Goodwin, L.; Startin, J. R.; Keely, B. J.; Goodall, D. M.; J. Chromatogr., A 2003 1004, 107.

24. Tadeo, J. L.; Sánchez-Brunete, C.; Pérez, R. A.; Fernández, M. D.; J. Chromatogr., A 2000, 882, 175.

25. Roy, D. N.; Konar, S. K.; Banerjee, S.; Charles, D. A.; Can. J. Forest. Res. 1989, 19, 842; Konar, S. K.; Roy, D. N.; Anal. Chim. Acta 1990, 229, 277.

26. Seiber, J. N.; Mcchesney, M. M.; Kon, R.; Leavitt, R. A.; J. Agric. Food Chem. 1984, 32, 678.

27. Guinivan, R. A.; Thompson, N. P.; Wheeler, W. B.; J. Assoc. Off. Anal. Chem. 1991, 74, 842.

28. Brady, N. C.; Natureza e propriedades dos solos, $6^{\mathrm{a}}$ ed., Livraria Freitas Bastos S.A.: Rio de Janeiro, 1983.

29. Malavolta, E.; Kliemann, H. J.; Desordens nutricionais no cerrado, POTAFOS: Piracicaba, 1985.

30. Mothci, E. P.; Amaral, J. A. M.; Santos, R. D.; Levantamento de reconhecimento-detalhado e aptidão agrícola dos solos da área do centro Nacional de Pesquisa de Gado de Corte, Mato Grosso do Sul, EMBRAPA/ SNLCS: Rio de Janeiro, 1979.

31. Morillo, E.; Undabeytia, T.; Maqueda, C.; Ramos, A.; Chemosphere 2000, 40, 103; Morillo, E.; Undabeytia, T.; Maqueda, C.; Ramos, A.; Chemosphere 2002, 47, 747.

32. Prata, F.; Cardinali, V. C. B.; Lavorenti, A.; Sci. Agric. 2003, 60, 175. 